\title{
The outer evolution of instability-generated structure in radiatively driven stellar winds
}

\author{
M. C. Runacres ${ }^{1}$ and S. P. Owocki ${ }^{2}$ \\ 1 Royal Observatory of Belgium, Ringlaan 3, 1180 Brussel, Belgium \\ 2 Bartol Research Institute, University of Delaware, Newark, DE 19716, USA
}

Received 7 August 2001 / Accepted 29 October 2001

\begin{abstract}
We investigate stochastic structure in hot-star winds. The structure (i.e. inhomogeneities such as clumps and shocks) is generated by the instability of the line driving mechanism in the inner wind. It is self-excited in the sense that it persists even in the absence of explicit perturbations. The evolution of structure as it moves out with the flow is quantified by the radial dependence of statistical properties such as the clumping factor and the velocity dispersion. We find that structure evolves under the influence of two competing mechanisms. Dense clumps pressure-expand into the rarefied gas that separates them, but this expansion is counteracted by supersonic collisions among the clumps, which tend to compress them further. Because of such ongoing collisions, clumps can survive over an extended region out of pressure equilibrium with the rarefied surrounding gas. Moreover, the linedriving force has little rôle in maintaining the structure beyond about $20-30 R_{*}$, implying that the outer evolution can be simplified as a pure gasdynamical problem. In modelling the distant wind structure we find it is necessary to maintain a relatively fine constant grid spacing to resolve the often quite narrow dense clumps. We also find that variations in the heating and cooling, particularly the "floor" temperature to which shock-compressed gas is allowed to cool, can affect both the density and temperature variation. Finally, we find that increasing the value of the line-driving cut-off parameter $\kappa_{\max }$ can significantly enhance the level of flow structure. Overall, the results of our work suggest that structure initiated in the inner wind acceleration region can survive to substantial distances $\left(\sim 100 R_{*}\right)$, and thus can have an important influence on observational diagnostics (e.g. infrared and radio emission) formed in the outer wind.
\end{abstract}

Key words. stars: early-type - stars: mass-loss - stars: winds, outflows - hydrodynamics - instabilities

\section{Introduction}

Hot stars of spectral type $\mathrm{O}$ and $\mathrm{B}$ have strong stellar winds, driven by the scattering of stellar continuum radiation in spectral lines. Line driving is also the most likely acceleration mechanism for winds from Wolf-Rayet stars, but as yet the hydrodynamics of these dense winds is not well understood. In this paper, we therefore limit ourselves to OB-star winds.

Three essential ingredients of line driving are resonant amplification, self-shadowing, and the Doppler effect. Resonant amplification causes the cross-section of bound electrons to be many orders of magnitude larger than the Thomson cross-section of free electrons (Heitler 1954; Gayley 1995). Even accounting for their relative scarcity with respect to free electrons, bound electrons are thus capable of providing a much larger acceleration than what is

Send offprint requests to: M. C. Runacres, e-mail: Mark.Runacres@oma.be needed to overcome the effective gravity. Self-shadowing of the scattering ions, however, blocks most of the flux available within the width of the spectral line and hence greatly reduces the magnitude of the line-driving force, bringing it back below the gravitational force.

The enormous potential of line driving would therefore remain untapped, were it not for the third ingredient, the Doppler effect. The motion of an ion away from the star allows unattenuated stellar flux to be red-shifted back into the line profile and increases the radiative force to which the ions are exposed. Due to Coulomb interactions the accelerated minor ions drag the bulk mass of hydrogen and helium ions with them. This brings the line driving force up to a level that is sufficient to overcome the inward pull of gravity and maintain a stellar wind. Line-scattering as the driving force of hot-star winds was first suggested by Lucy \& Solomon (1970). A formalism to quantitatively model line-driven hot-star winds was introduced by Castor et al. (1975, hereafter CAK), and 
developed further (e.g. to account for the finite angle of the stellar core) by Pauldrach et al. (1986) and Friend \& Abbott (1986). Gayley (1995) introduced an improved formalism that greatly helps clarify the physical interpretation of the line opacity and associated driving.

Long before being actually applied to hot-star winds, it was already realised (Milne 1926) that line driving is highly unstable. The reason for the instability is again the Doppler effect: a small increase in velocity of a fluid element exposes the element to fiercer radiation from the star and causes it to be further accelerated (MacGregor et al. 1979; Owocki \& Rybicki 1984, 1985). Time-dependent hydrodynamical simulations of unstable line-driven stellar winds were first performed by Owocki et al. (1988, hereafter OCR), and later by Feldmeier (1995), Feldmeier et al. (1997b) and Owocki \& Puls (1996, 1999, hereafter OP96 and OP99, respectively). All these simulations agree on the main features of instability-generated structure: the wind is pervaded by strong shocks (reverse and forward) that can heat the gas to temperatures in excess of a million degrees and compress it into clumps that are typically an order of magnitude denser than the ambient wind.

The instability-generated structure is stochastic in nature and should not be confused with large-scale, localised structures such as co-rotating interaction regions (CIRs). These CIRs are thought to cause the rotational modulation of discrete absorption components seen in ultraviolet spectral lines of hot stars (Prinja 1998). The observational evidence for instability-generated structure is more indirect. Soft X-rays from hot stars, as well as the presence of high ionisation stages such as $\mathrm{N} \mathrm{V}$, point to shocks that could be instability-generated (Lucy 1982a). Furthermore, the extended black troughs of saturated ultraviolet lines are indicative of increased back-scattering associated with a non-monotonic velocity (Lucy 1982b). Finally, the nonthermal radio emission detected for some of the brightest O-stars (Bieging et al. 1989) is thought to be synchrotron emission from shock-accelerated particles (Chen \& White 1994).

Most previous papers on instability-generated wind structure focus on the inner or intermediate wind (below 30 stellar radii). There are however numerous reasons to study structure in the outer wind. One of the most reliable derivations of the mass-loss rates of hot stars is from the thermal radio continuum due to free-free emission (Bremsstrahlung). The conversion of a radio flux into a mass loss rate (Wright \& Barlow 1975) depends on the degree of clumping of the gas (Abbott et al. 1981). Underestimating the degree of clumping results in an overestimate of the mass-loss rate.

The synchrotron radiation that is thought to be responsible for non-thermal radio emission has to originate beyond $\sim 100 R_{*}$, as photons originating closer to the stellar surface would be shielded by the large free-free opacity of the gas. Furthermore, the shock-acceleration itself has to happen in situ, as Compton cooling prevents relativistic particles from travelling large distances (Chen \& White 1994). This points to the existence of shocks at large distances from the star. Inverse Compton emission by relativistic particles produces non-thermal X-rays, and from the comparison of the non-thermal X-ray and radio emission one can in principle derive the magnetic field strength (Pollock 1987).

The formation region of thermal X-rays depends sensitively on the recombination of fully ionised helium to $\mathrm{He}^{+}$ (Hillier et al. 1993; Feldmeier et al. 1997a). In winds where He recombines, observable X-rays must be emitted more than a hundred stellar radii above the stellar surface.

Finally, many Wolf-Rayet stars are surrounded by ringnebulae. These nebulae are formed when a fast wind overtakes a slower wind from a previous evolutionary stage. High-resolution images have shown these nebulae to be highly structured. This structure can be caused by instabilities associated with the wind-interaction or could also be an imprint of structure in the wind itself (Grosdidier et al. 1998). This would require structure to persist up to very large distances from the star.

This paper presents radiation hydrodynamical simulations of wind structure out to distances of 100 stellar radii from the stellar surface. In Sect. 2 we discuss the general principles of our models. In Sect. 3 we give the statistical quantities which describe the wind structure. In Sect. 4 we present the reference model, with which all the other models are compared. In the next sections we discuss the effect of various processes and assumptions on the outer-wind structure: external forces such as line driving (Sect. 5), the spacing of the radial mesh (Sect. 6), the line-strength cut-off parameter (Sect. 7) and heating and cooling by radiation (Sect. 8). Conclusions are presented in Sect. 9.

\section{General description of models}

\subsection{Conservation equations}

Consider an inviscid flow along the radial direction $r$ from a central star. In Eulerian form, the one-dimensional (1D) time-dependent equations for conservation of mass, momentum, and energy are:

$$
\begin{aligned}
& \frac{\partial \rho}{\partial t}+\frac{1}{r^{2}} \frac{\partial\left(r^{2} \rho v\right)}{\partial r}=0 \\
& \frac{\partial(\rho v)}{\partial t}+\frac{1}{r^{2}} \frac{\partial\left(r^{2} \rho v^{2}\right)}{\partial r}=-\frac{\partial p}{\partial r}-\rho g_{*}+\rho g_{\mathrm{rad}} \\
& \frac{\partial e}{\partial t}+\frac{1}{r^{2}} \frac{\partial\left(r^{2} e v\right)}{\partial r}=-\frac{p}{r^{2}} \frac{\partial\left(r^{2} v\right)}{\partial r}-Q_{\mathrm{rad}}
\end{aligned}
$$

Here $e$ is the internal energy density (in $\mathrm{erg} / \mathrm{cm}^{3}$ ), $g_{*}$ the effective stellar gravity (reduced by electron scattering), $g_{\mathrm{rad}}$ the acceleration due to line driving and $Q_{\mathrm{rad}}$ the power emitted by radiation per unit volume. The other symbols have their usual meaning. The set of equations is supplemented by the perfect gas law.

Since both the radiative driving and its associated instability are strongest in the radial direction 
(Rybicki et al. 1990), the assumption here of an effectively 1D, radial flow represents a reasonable first approximation. But more generally, it seems quite likely that the lateral coherence of structure generated by the radial, linedriven instabilities would be disrupted by other, lateral instabilities (e.g., Rayleigh-Taylor) that require a more complete multidimensional treatment. Unfortunately, the non-local integrations required for computation of the line-force become quite formidable to carry out in more than 1D, and as such, there have so far been only some quite limited explorations of the possible multidimensional nature of the structure arising from the line-driven instability (Owocki 1999). In this sense, the 1D simulations here can be thought as modelling the flow dynamics in some typical solid-angle patch of the overall spherical outflow, with the lateral coherence size of this patch yet to be determined (Dessart \& Owocki 2001).

The conservation Eqs. (1)-(3) are solved using VH1 , a numerical code developed by J. M. Blondin and colleagues (Blondin, personal communication), by explicitly time-stepping the Lagrangian form of these equations and then remapping the evolved hydrodynamic variables back onto a fixed Eulerian grid after every time-step. In both the Lagrangian update and the remap, the Piecewise Parabolic Method (PPM; Colella \& Woodward 1984) is used.

\subsection{Line driving}

The forces in the momentum equation are due to the pressure gradient, gravity and line driving. This last force is the most important in two ways: it is the force that drives the wind and its evaluation is what dominates the computing time of the simulation. In this paper, it is evaluated using the smooth source function (SSF) method (Owocki 1991). A more sophisticated approach (EISF, for "escapeintegral source function") exists, but is computationally much more expensive and leads to structure that is qualitatively similar to the results of the cheaper SSF method (OP99).

The force from all lines together is obtained as the integral of the single-line force over a spectrum-averaged line-number distribution

$$
\frac{\mathrm{d} N(\kappa)}{\mathrm{d} \kappa}=\frac{1}{\kappa_{0}}\left(\frac{\kappa}{\kappa_{0}}\right)^{\alpha-2} \mathrm{e}^{-\kappa / \kappa_{\max }} .
$$

The normalisation constant $\kappa_{0}$ is related to the strength of the strongest line. The exponent $\alpha$ can be interpreted as the ratio of the force from optically thick lines to the total line force. The cut-off parameter $\kappa_{\max }$ limits the maximum line strength (see Sect. 7). Although the CAK line-force assumes $\kappa_{\max } \rightarrow \infty$, its implicit assumption of a continuous power-law distribution breaks down for the discrete number of strong lines, i.e. when $\kappa \mathrm{d} N / \mathrm{d} \kappa \approx 1$. More realistically, one should thus generally impose an opacity cut-off with $\kappa_{\max } \approx \kappa_{0}$.

For sufficiently thick wids, the above parametrisation leads to a force that is proportional to $\tau_{0}^{-\alpha}$, where $\tau_{0}$ is the optical thickness for a single line of opacity $\kappa_{0}$. For winds that are optically thin in all lines the force no longer increases with decreasing optical depth (Abbott 1982).

For the above line distribution, OP96 derive from first principles the expressions for the line force in the SSF approximation, giving a full discussion of the underlying assumptions.

\subsection{Cooling and heating}

In the energy equation, the two terms on the right-handside represent adiabatic and radiative cooling. The power radiated per unit volume can be written as

$Q_{\mathrm{rad}}=n_{\mathrm{e}} N_{\mathrm{H}} \Lambda(T)$

where $n_{\mathrm{e}}$ and $N_{\mathrm{H}}$ respectively represent the number density of electrons and hydrogen (atoms and ions), and the cooling coefficient $\Lambda$ describes the temperature dependence of the cooling (Raymond et al. 1976).

The combined effect of this cooling is balanced by photoionisation heating from the star's UV radiation, which in a relatively smooth, steady outflow has the general effect of thus preventing the wind from cooling much below the stellar effective temperature (e.g., Drew 1989). In this paper, we mimic this effect of photoionisation heating by simply requiring that the wind temperature never fall below a floor value, typically given by the stellar effective temperature. A more complete treatment of such heating in the kind of highly structured, time-dependent flows modelled here is beyond the current scope, and so will be deferred to future study.

Indeed in the structured models here, adiabatic compression and shock conversion of flow kinetic energy often lead to regions of strong compressive heating, which are then subsequently cooled by the radiative emission, as modelled by the cooling function $\Lambda$. Feldmeier (1995) describes how numerical hydrodynamical flows with such cooling are subject to various effects that cause the cooling zone to effectively collapse, thus leading to an underresolution that causes the amount of hot gas to be substantially underestimated. In an effort to identify sufficient hot material to explain observed levels of hot-star X-ray emission, Feldmeier (1995) artificially limited the slope of the cooling function in a way that partially mitigates this underresolution. In the present context, resolving the hot gas is less vital, and we have opted to retain a tabulated cooling function without such modifications. Moreover, our use here of a constant, relatively fine spatial grid means that such underresolution is less severe (see Fig. 1) than in previous models with grid spacing that increases with radius.

\subsection{Boundary and initial conditions}

The simulation volume extends from $r=1 R_{*}$, the photospheric radius where the optical depth for electron scattering is approximately one, to an outer radius 
$r=R_{\max }=101 R_{*}$. At the inner boundary we fix the density $\rho$ and the temperature $T$ but allow the velocity $v$ to float, with the requirement that its second derivative be zero. At the outer boundary, both $\rho$ and $v$ may vary, but must have zero second derivatives, while $T$ is taken constant. Unlike some previous simulations, we do not explicitly perturb the base of the stellar wind by a sound wave or turbulence. Nonetheless, we find the flow in the outer wind develops a persistent, "intrinsic" variability. As discussed by OP99, such self-excitation of intrinsic variability in the absence of any explicit perturbations has its physical origin in the backscattering of radiation from outer wind structure, which thus seeds small variations at the wind base that subsequently become amplified to maintain the strong structure of the outer wind. The exact reason why self-excited structure is stochastic in nature, and not e.g. quasi-periodic, is not fully understood and requires further study.

Each simulation starts from a smooth wind initial condition computed from a CAK/Sobolev approximation for the line-force. It is then run using the SSF line-force for $2 \mathrm{Msec}$ to allow the response to the initial condition to die away (the time needed to cross the simulation volume at $2000 \mathrm{~km} \mathrm{~s}^{-1}$ is $\left.\sim 0.7 \mathrm{Msec}\right)$. The model is then evolved for detailed study over a further $500 \mathrm{ksec}$. Time-averaged quantities needed for the statistical description of structure (Sect. 3) are calculated by summing the relevant variables at every time step over this $500 \mathrm{ksec}$ interval.

A further important detail is that the time evolution is computed with a fixed time-step, rather than using a time-step that varies to satisfy a fixed Courant number (see, e.g., Laney 1998). The latter approach can introduce an additional, artificial feedback between outer and inner wind structure. (See, e.g., discussion surrounding Eq. (31) of Poe et al. 1990.) Our choice of a fixed time-step of $5 \mathrm{~s}$ satisfies the Courant condition with a Courant number never exceeding 0.5 .

\section{Statistical description of structure}

The evolution of structure as it moves out with the flow can be usefully characterised by a number of statistical quantities. In this paper we mainly use the clumping factor, the velocity dispersion and the correlation coefficient, defined as

$$
\begin{aligned}
& f_{\mathrm{cl}}=\frac{<\rho^{2}>}{<\rho\rangle^{2}} \\
& v_{\mathrm{disp}}=\sqrt{<v^{2}>-<v>^{2}} \text { and } \\
& C_{v \log \rho}=\frac{<v \log \rho>-<v><\log \rho>}{v_{\operatorname{disp}} \sqrt{<(\log \rho)^{2}>-<\log \rho>^{2}}},
\end{aligned}
$$

respectively. The symbol $<>$ denotes a time average and all of the above quantities may depend on radius.

The clumping factor describes how mass is distributed; it becomes larger as mass is concentrated in a smaller volume. The velocity dispersion is the standard deviation of the velocity and as such describes the typical velocity variation around the mean flow speed. The correlation coefficient gives the correlation between density and velocity variations and has values between -1 (complete anticorrelation) and 1 (complete correlation, i.e. velocity and density are in phase). Positive (negative) correlation indicates a net preponderance of structure that propagates outward (inward) relative to the mean outflow. In particular, for variations due to shocks, a positive (negative) correlation indicates a net preponderance of forward (reverse) shocks.

Diagnostics sensitive to the square of the density (e.g., emission in Balmer lines or the infrared and radio continua) will tend to overestimate the mass loss rate of a clumped wind by a factor of $\sqrt{f_{\mathrm{cl}}}$. In this context, an alternative quantity commonly used to describe wind structure is the volume filling factor $f$. It is defined as the fractional volume of the dense gas, assuming that the gas is made up of two components: dense clumps and rarefied interclump material. For a constant filling factor, the clumping factor can then be expressed (Abbott et al. 1981) as

$f_{\mathrm{cl}}=\frac{f+(1-f) x^{2}}{[f+x(1-f)]^{2}}$,

where $x$ is the ratio of low to high density gas. If most of the mass is concentrated in the clumps $(f \gg(1-f) x)$, the clumping factor is the inverse of the volume filling factor, and equals the density contrast of the clumps with respect to the mean density.

\section{Reference model}

The stellar parameters and wind parameters used for the reference model are the same as those in OP99, and are given in Table 1. Although not specifically intended to model a particular star, the stellar parameters correspond to an early $\mathrm{O}$ supergiant such as $\zeta$ Pup. The chosen parameters result in a mass loss rate of $3.5 \times 10^{-6} M_{\odot} / \mathrm{yr}$ and a terminal speed of $2000 \mathrm{~km} \mathrm{~s}^{-1}$. It is assumed that $\mathrm{H}$ and $\mathrm{He}$ are fully ionised.

We use a fixed spatial mesh of 10637 points. In order to adequately resolve the region of rapid acceleration, the initial 1025 points have a spacing that increases linearly from 0.001 to $0.01 R_{*}$ over the radius range $r=1-5 R_{*}$. The remaining points use a constant spacing of $0.01 R_{*}$ spanning the range $r=5-101 R_{*}$.

\subsection{Wind structure}

Figures 1 and 2 show snapshots of the radial variation of velocity, density and temperature in the inner and outer wind. Many of the features have been extensively discussed in other papers (e.g. OCR, Feldmeier 1995; Feldmeier et al. 1997b, OP99), and so here we focus mainly on the radial evolution of the overall structure properties.

The wind can be divided into a number of geometric regions for which the boundaries are somewhat fuzzy, but which nevertheless have their own typical characteristics. 
Table 1. Stellar parameters and wind parameters for the reference model.

\begin{tabular}{lll}
\hline quantity & symbol & value \\
\hline stellar mass & $M$ & $40 M_{\odot}$ \\
photospheric radius & $R_{*}$ & $19 R_{\odot}$ \\
effective temperature & $T_{\text {eff }}$ & $37800 \mathrm{~K}$ \\
CAK exponent & $\alpha$ & 0.7 \\
opacity constant & $\kappa_{0} v_{\text {th }} / c$ & $3500 \mathrm{~cm}^{2} / \mathrm{g}$ \\
line strength cut-off & $\kappa_{\max }$ & $0.001 \kappa_{0}$ \\
H abundance by mass & $X$ & 0.73 \\
He abundance by mass & $Y$ & $1-X$ \\
thermal speed & $v_{\text {th }}$ & $0.28 a^{\dagger}$ \\
\hline
\end{tabular}

$\dagger$ Isothermal sound speed $a=\sqrt{p / \rho}$.

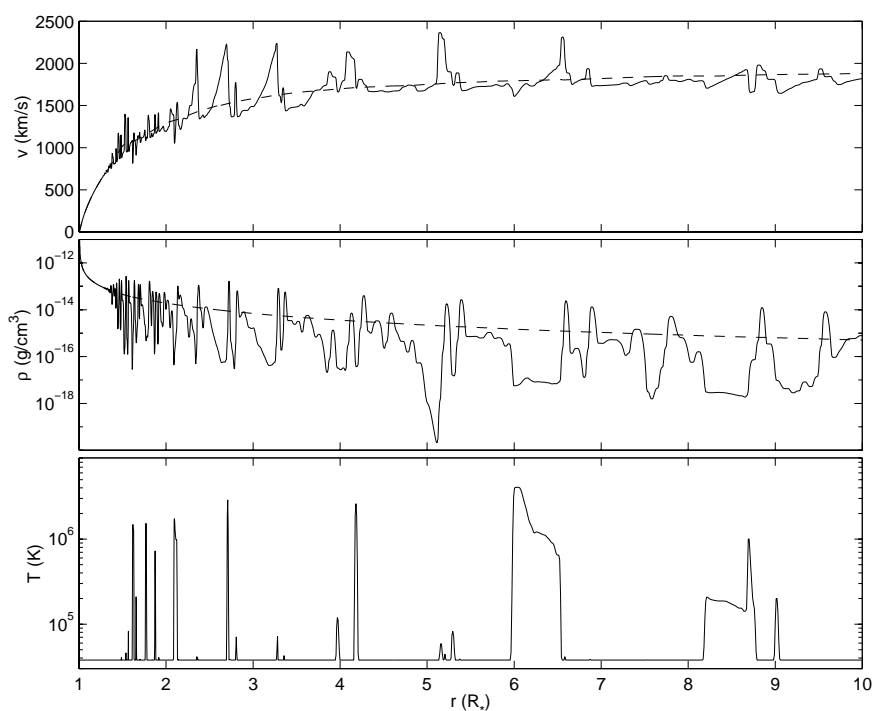

Fig. 1. Snapshot of the reference model in the inner wind, at 2.0 Msec after the start of the simulation. The dashed line in the upper panels represents time-averaged values.

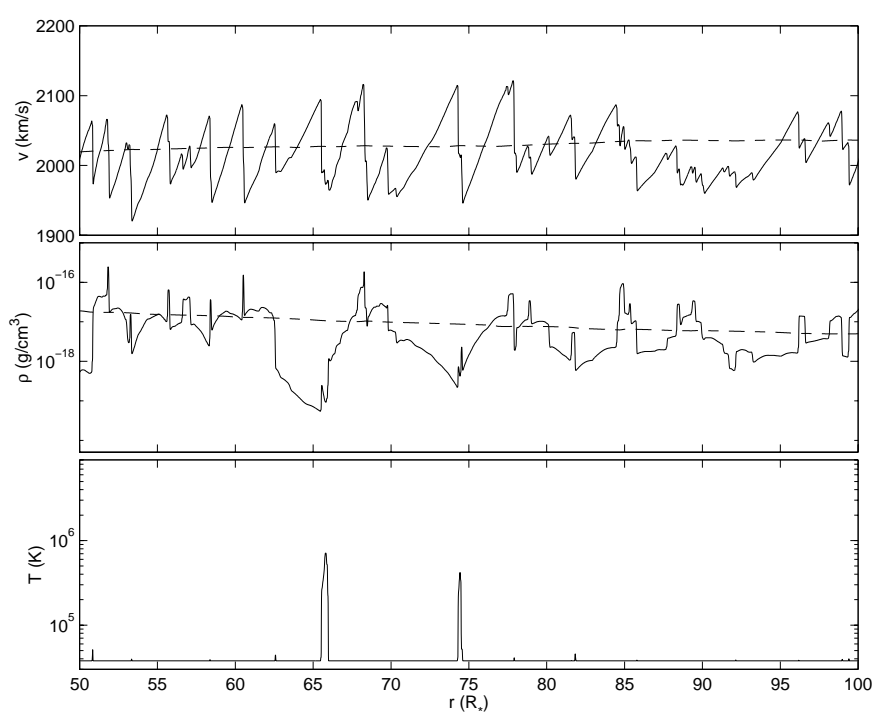

Fig. 2. Same as Fig. 1, but for a representative portion of the outer wind. Note that the range in radius is larger than on the previous figure.

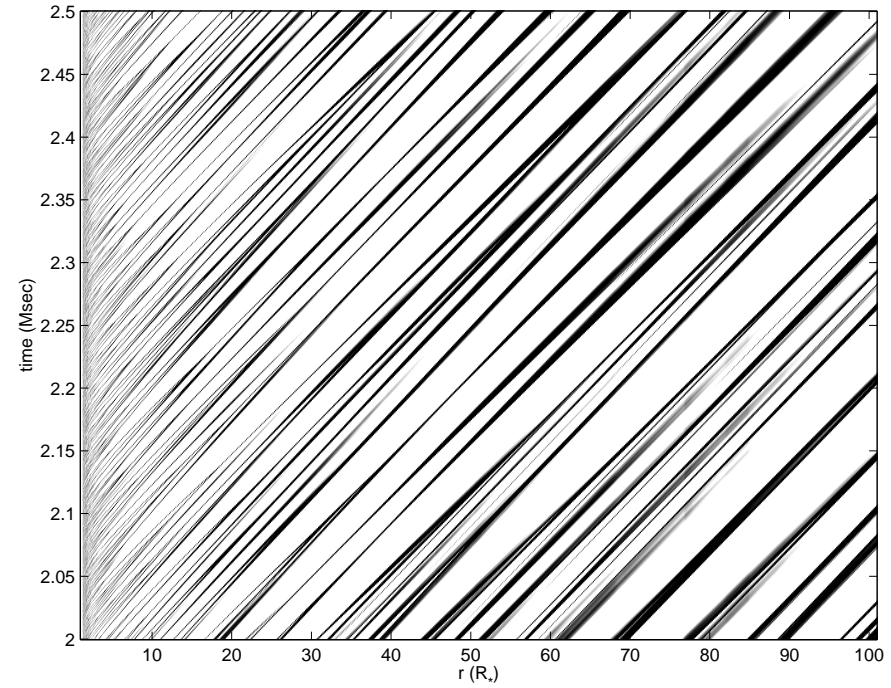

Fig. 3. Spatial and temporal evolution of the normalised density $\rho /\langle\rho\rangle$. To highlight the dense clumps, the lower and upper cut-offs of the grey-scale have been set to 1 and 2 , respectively; gas with a density below the mean appears as white, and all shells with a density larger than $2<\rho\rangle$ as black.

The outflow at the base of the wind is almost steady. But starting at $r \sim 1.3 R_{*}$ irregular variations appear in the velocity and density. The variations rapidly steepen into shocks (for a detailed discussion of the onset of structure in SSF models, see OP99). These initial shocks are reverse type, which means that they propagate inward relative to the gas, although the overall outflow advects them outward relative to the star. They decelerate and compress rarefied gas that has been accelerated to high-speed by the instability. Most of the velocity peaks in the upper panel of Fig. 1 are steep rarefaction waves terminated by a reverse shock.

In this initial structure, most of the stellar wind material thus becomes collected into in a sequence of dense clumps bounded on the inside by a reverse shock that separates the clumps from the much more rarefied, high speed flow in between them. After a few stellar radii many clumps also become bounded on the outside by a weaker forward shock, whenever clumps flow faster than material ahead of them. For example, a weak forward shock is visible around $5.3 R_{*}$, just beyond a strong reverse shock. The structure then finds its definite form: a sequence of dense clumps bounded on both sides by shocks that feed rarefied gas into the clumps. Most of the shocks in Fig. 2 occur in such reverse-forward pairs.

The clumps are typically an order of magnitude denser than the average wind. They move at approximately the terminal velocity but have finite relative velocities, causing them to collide and form denser clumps. This can be seen from Fig. 3, which shows the density (normalised to the mean density $\langle\rho\rangle$ ) as a function of radius and time, with the dark streaks indicating the motion of a clump. The importance of clump collisions for X-ray production has been emphasised by Feldmeier et al. (1997b). In the 

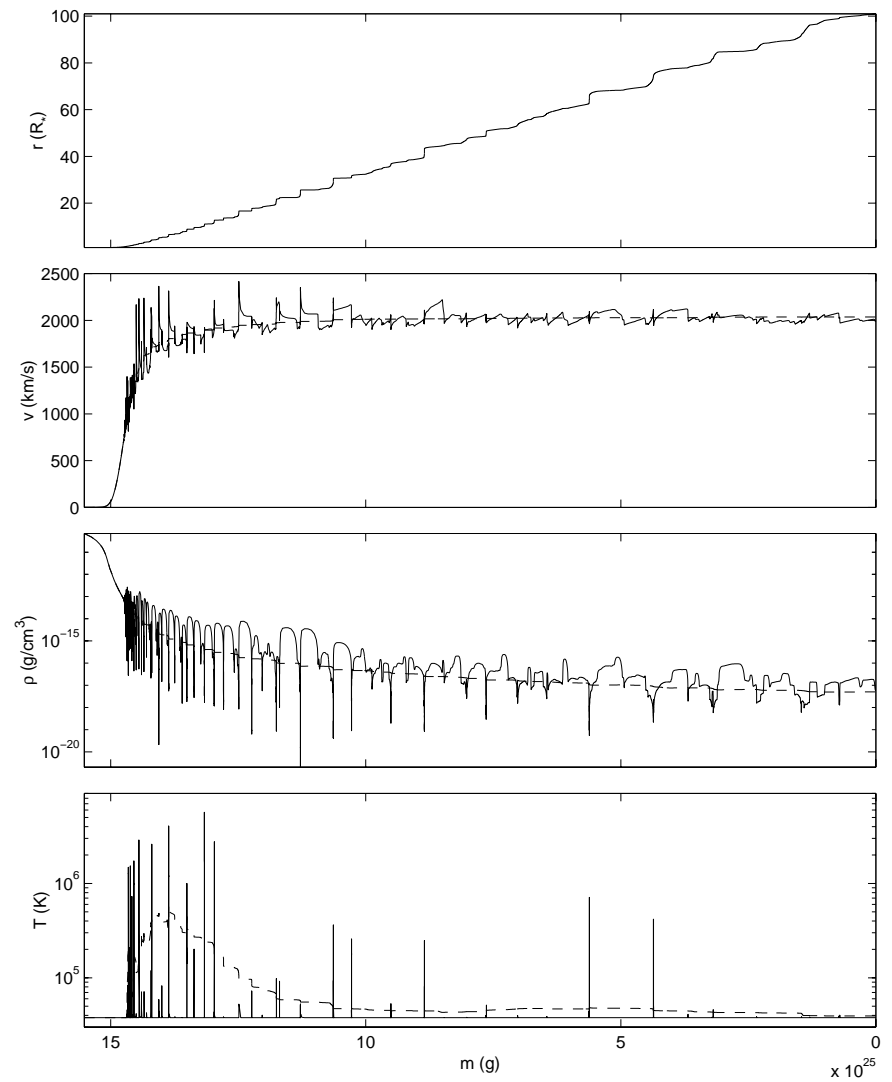

Fig. 4. Snapshot of the reference model at $2 \mathrm{Msec}$, now plotted versus the Lagrangian mass coordinate $m$ defined in Eq. (10). The upper panel shows the Eulerian radius, while the remaining panels show the velocity, density, and temperature. The dashed lines in these lower panels show the corresponding timeaveraged values.

simulations by these authors, the base of the wind was perturbed by a sound wave or turbulence. Our results show that clump collisions can also occur when the structure is self-excited, although generally with lower relative speeds than in the perturbed models. As they result in denser clumps, collisions also play an important rôle in maintaining structure. Note moreover that collisions can persist to quite large distances from the star.

Fig. 4 shows a wind snapshot at 2 Msec plotted versus a Lagrangian mass coordinate (OCR, OP99) defined by

$m(r) \equiv \int_{r}^{R_{\max }} 4 \pi \rho\left(r^{\prime}\right) r^{\prime 2} \mathrm{~d} r^{\prime}$.

Such a plot emphasises the relative amount of mass to be associated with various flow structures. In particular, note that the high-speed rarefactions that form from the initial instability actually contain very little mass, and so are not likely to have much direct effect on, e.g., observable line-profile variations. Likewise, the lowermost panel makes clear that there is really very little material that is shock-heated to temperatures substantially above the wind floor temperature. As such, these models of intrinsic variability generally yield far less X-ray emission than is commonly observed for hot-star winds. Moreover, this plot

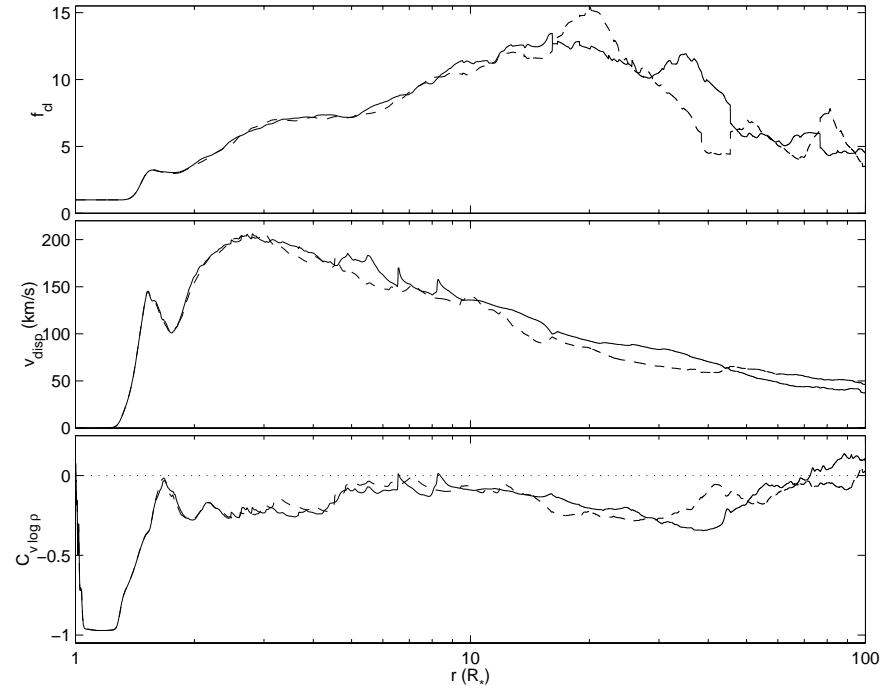

Fig. 5. Statistical properties of the reference model. The three panels, from top to bottom, show the clumping factor, the velocity dispersion, and the velocity-density correlation, all as a function of radius. The full line corresponds to averages taken between 2 and $2.5 \mathrm{Msec}$, the dashed line to averages taken between 2.5 and 3 Msec. The zero level for the correlation function is indicated by a dotted line.

shows that the purely spatial average of the temperature given by the dashed curve is actually not very representative of the temperature for most of the wind mass.

On the other hand, the mass plots of velocity and density illustrate quite well the persistence of substantial velocity dispersion and clumping through an extended range of material in the outer wind.

\subsection{Statistical properties}

The statistical properties of the reference model (Fig. 5) also reflect the distinct characteristics of the different stellar wind regions. To judge the influence of the time interval over which the statistical quantities are calculated, we continued the reference model for another $500 \mathrm{ksec}$, i.e. over 2.5-3 Msec. Quantities for the standard model (from 2 to $2.5 \mathrm{Msec}$ ) are indicated by solid lines, and those for the $500 \mathrm{ksec}$ extension by dashed lines. The good overall agreement between these indicates that the statistical quantities do not depend much on the integration interval and so are intrinsic properties of the model.

At the base of the wind (below $r \sim 1.5 R_{*}$ ), the mass distribution is smooth $\left(f_{\mathrm{cl}}=1\right)$ and the variations in the velocity are extremely small $\left(v_{\text {disp }} \ll a\right)$. As is typical for SSF calculations (OP99), structure appears with almost perfectly anti-correlated variations of density and velocity $\left(C_{\mathrm{v} \rho} \approx-1\right)$. These variations grow dramatically and steepen into shocks. The subsequent nonlinear interaction from clump collisions quickly disrupts this flow anti-correlation, so that above $r \approx 2 R_{*}$ there is little net velocity-density correlation, indicating there is roughly equal mixture of forward and reverse propagating structure. 


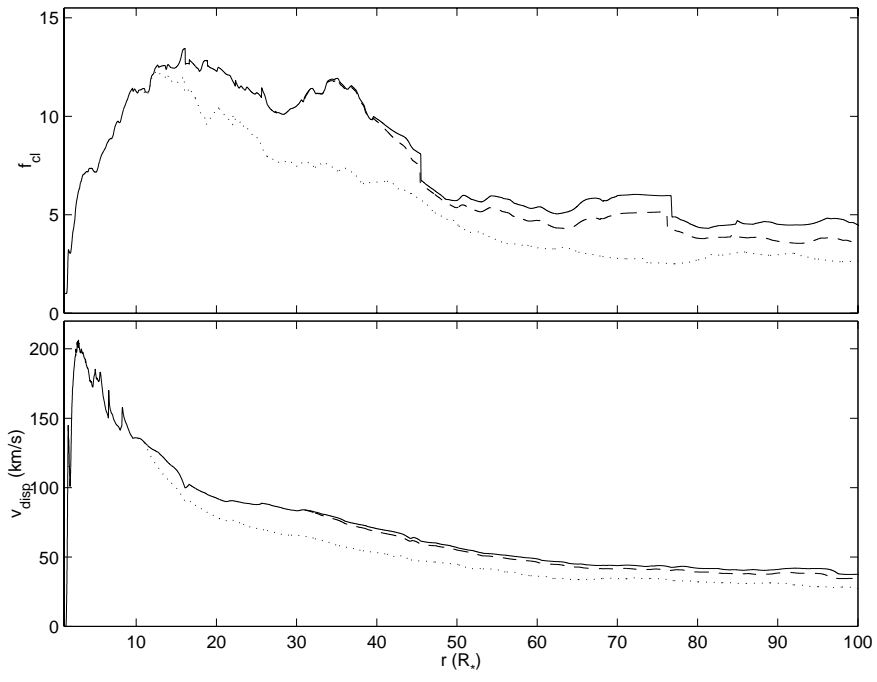

Fig. 6. Statistical properties of the reference model (solid line), compared to two models where the external forces have been set to zero beyond $11 R_{*}$ (dotted line) and $31 R_{*}$ (dashed line).

The steep initial rise in velocity dispersion reflects the initial strong amplification of velocity variations by the line-driven instability. This initial rise is temporarily halted as the high-speed rarefactions are filled in and the anti-correlation vanishes. But then, quite surprisingly, there develops a second rise in dispersion, characterised now by little net correlation between velocity and density. Moreover, even after the dispersion reaches an absolute maximum rms amplitude of $\sim 200 \mathrm{~km} \mathrm{~s}^{-1}$ at around $r \approx 3 R_{*}$, the subsequent decline is quite gradual, with a residual dispersion of $\sim 50 \mathrm{~km} \mathrm{~s}^{-1}$ persisting even at $r=100 R_{*}$.

Perhaps even more surprising, the density clumping factor actually continues to rise out to nearly $r \approx 20 R_{*}$. The supersonic collisions among the clumps tend to compress them further, causing a steady rise in the clumping factor. But as these collisions become weaker and less frequent in the outer wind, the pressure-driven expansion of individual clumps into the rarefied regions between them eventually causes the overall clumping factor to slowly decline.

One simple way to maintain structure up to large distances would be to have cold, high density gas in pressure equilibrium with hot, rarefied gas. Shocks do heat some gas to very high temperatures, but due to the efficiency of radiative cooling, only the most rarefied gas can remain hot. It is, however, not hot enough to balance the pressure of the dense clumps. It appears therefore, that under the present assumptions, clump collisions are the key mechanism to maintain a structured wind.

\section{The effect of external forces at large distances}

As both gravity and the line driving force fall off as $1 / r^{2}$ at large distances, where the star appears as a point source, we can expect them to become unimportant far away from

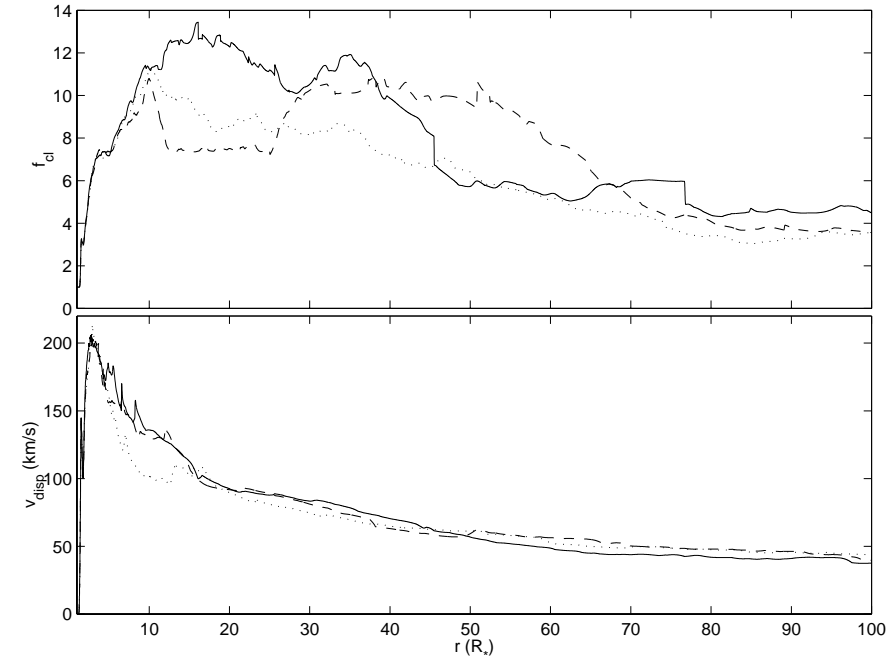

Fig. 7. Influence of the spatial grid on the statistical properties of the model. The solid line corresponds to the reference grid, the dashed line to the grid with doubled spacing and the dotted line to a grid with spacing proportional to $r$.

the star. In the reference model, the time-averaged wind has reached $92 \%$ of its terminal velocity at $10 R_{*}$, suggesting a minor effect of external forces beyond that distance. It is not clear, however, to what extent this estimate pertains to the formation of structure. To examine the rôle of external forces on the outer wind variability, we now compare our full results with simulations that are assumed to be "force-free", i.e. no gravity or radiative force, beyond a certain distance.

To do this, we stored the time-series of the density, velocity and pressure from the reference model at some intermediate radius $R_{\text {in }}$ and used this time-series as a fixed inflow inner boundary condition for the force-free model that extends from $R_{\text {in }}$ to the usual outer boundary $R_{\max }=$ $101 R_{*}$. We use two different values for $R_{\text {in }}: 11$ and $31 R_{*}$. Again, care was taken to run the model for a sufficiently long time, to allow the response to the initial condition to die away.

Figure 6 shows the statistical properties of the reference model (solid line), and two force-free models with $R_{\text {in }}=11 R_{*}$ (dotted line), $R_{\text {in }}=31 R_{*}$ (dashed). The statistical properties of the $R_{\text {in }}=11 R_{*}$ model differ substantially from the reference model, indicating that, even though the wind has nearly reached its terminal velocity, the driving force still plays a rôle in the formation of structure. Beyond $31 R_{*}$, this is no longer the case and the statistical properties of the force-free model are essentially the same as for the reference model.

This implies that the outer wind evolution beyond $r \approx 30 R_{*}$ is a pure gasdynamical problem, for which radiation driving merely sets the inner boundary condition. In related work, we have utilised this principle to develop "pseudo-periodic" models that can be calculated out to very large distances (i.e. $1000 R_{*}$ ) at relatively low cost, by repeating a fixed time-series at an inner boundary 
set at an intermediate radius $R_{\text {in }} \approx 30 R_{*}$. (For an initial discussion, see Owocki et al. 2000.)

\section{The influence of the spatial grid}

Let us next investigate how the evolution of structure may be affected by the spatial mesh used in the simulations. We compare the reference grid (Sect. 4) with two modified grids: one with double the original grid spacing and one with a grid spacing increasing linearly as a function of radius, as used e.g. by Feldmeier et al. (1997b). As we are interested in the effect of grid spacing on the outer wind evolution, we keep the grid below $10 R_{*}$ unchanged. To avoid a discontinuity in the mesh spacing, the doubling is implemented over a transition region between 10 and $12 R_{*}$, where $\Delta r$ increases linearly from 0.01 to $0.02 R_{*}$. For the second grid, the grid spacing increases from $0.01 R_{*}$ at $11 R_{*}$ to $0.1 R_{*}$ at $R_{\max }$. For both models, the time averages were again calculated over a $500 \mathrm{ksec}$ interval after $2 \mathrm{Msec}$ to allow the response to the initial condition to die away.

In Fig. 7 we show the influence of the spatial grid on the clumping factor and velocity dispersion. The solid line corresponds to the reference grid, the dotted line to the grid with increasing step size, and the dashed line to the grid with doubled spacing. The most obvious difference is in the clumping factor. The strongest clumps in the reference model occur between 10 and $15 R_{*}$ as a result of collisions and are often as narrow as $0.05 R_{*}$. These clumps are smeared by the coarser grids. The agreement in the inner wind is much better, of course, but even there there are some minor differences, due to the backscattering effect mentioned previously.

The instability of line driving generates structure down to the smallest spatial scales of our radial mesh. In this sense, hydrodynamical simulations of line-driven flows are never truly grid-independent, as the grid sets an artificial limit to the resolution scale. As the instability growth rate is strongest for variations near and below the Sobolev length, the grid in the inner wind is typically chosen to resolve some fraction of this length (OCR). The results in this section indicate that for structure generated on a given inner wind grid, care must be taken in increasing the grid spacing in the outer wind, as features can remain surprisingly narrow up to quite large distances when adequately resolved.

\section{The importance of the line-strength cut-off}

As first noted by OCR, the high-speed rarefactions that arise in simulations of line-driven instability tend to continue to grow until they become optically thin to the strongest driving line. To keep such structure from becoming too steep to resolve with the assumed spatial grid, OCR introduced an opacity cut-off, which for relatively dense O-supergiant wind models had to be set to an artificially low value $\kappa_{\max } \approx 10^{-3} \kappa_{0}$. (As discussed in Sect. 2.2 , a realistic value is $\kappa_{\max } \approx \kappa_{0}$.) Such a low cut-off has since

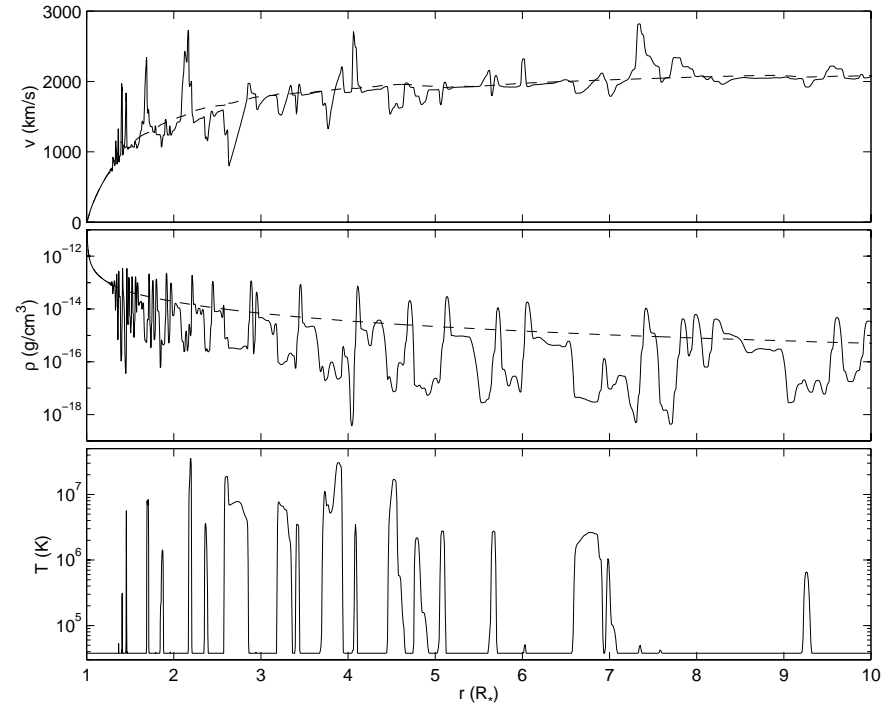

Fig. 8. Snapshot of the inner wind at 2.0 Msec after the start of the simulation, for the model with $\kappa_{\max }=10^{-2} \kappa_{0}$. The dashed line in the upper panels represents time-averaged values.

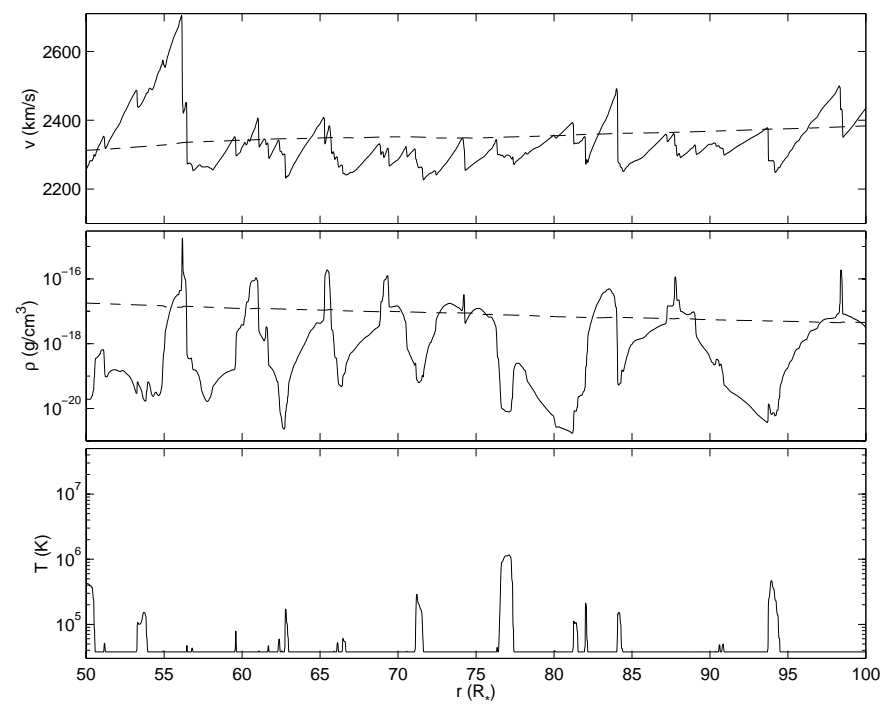

Fig. 9. Same as Fig. 8, but for the outer wind.

been used in essentially all instability simulations of dense winds, and so also provides the basis for the standard case presented above. But in experimentation we have done with relatively fine grid resolution, we have found it possible to increase this cut-off to less artificial levels. Here we present an initial comparison of models with different levels of this cut-off parameter.

Specifically, Figs. 8 and 9 show snapshots of a model where $\kappa_{\max }$ has been increased from $10^{-3} \kappa_{0}$ to $10^{-2} \kappa_{0}$. The effect of this increase is to include $\sim 10$ strong lines that were not present in the reference model. It takes steeper velocity gradients for these lines to become optically thin than for the strongest lines in the reference model. They thus allow for much stronger rarefactions and shocks. Some of the gas is heated to very high temperatures. Although this hot gas is still only a minute fraction of the wind mass (as it was for the reference model), 


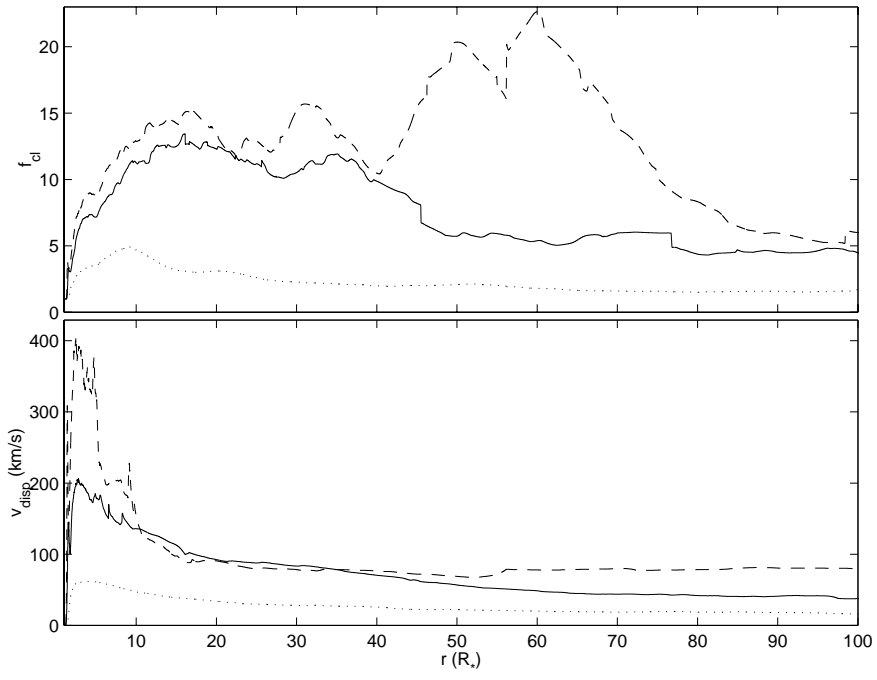

Fig. 10. Clumping factor and velocity dispersion for three different values of $\kappa_{\max }$. The solid line is the reference model, with $\kappa_{\max }=10^{-3} \kappa_{0}$. The dashed line corresponds to $\kappa_{\max }=$ $10^{-2} \kappa_{0}$, and the dotted line to $\kappa_{\max }=10^{-4} \kappa_{0}$.

increasing $\kappa_{\max }$ does increase the mass fraction of X-ray emitting material. (For the snapshot shown above, $0.12 \%$ of the wind mass is in excess of a million degrees, compared to $0.02 \%$ for the reference model.)

Figure 10 shows the statistical properties of the model with $\kappa_{\max }=10^{-2} \kappa_{0}$ (dashed line) and the reference model (solid line), together with a model where $\kappa_{\max }$ has been decreased by a factor of ten (dotted line). Reducing $\kappa_{\max }$ by this amount leaves only the weaker lines to drive the wind and very little structure is formed. The strongest clumping in the model with increased $\kappa_{\max }$ occurs very far from the star (at $60 R_{*}$ ) and is due to collisions forming dense and narrow clumps (with density enhancements reaching values over 100 and widths below $0.1 R_{*}$ ). Resolving these clumps requires a small grid spacing over almost the entire grid. Hardly any collisions happen beyond $60 R_{*}$ and at the outer boundary the clumping factor is almost the same as for the reference model.

The change in $\kappa_{\max }$ also has a modest effect (of the order of $10 \%$ ) on the mass loss rate and terminal velocity. This is due to the fact that with the smaller cut-off the distribution contains about $10 \%$ fewer optically thick lines. Accordingly, using the CAK/Sobolev line driving force in the point-star approximation as an estimate, we find the cut-off reduces the force by about $10 \%$ (we find a value of 0.9 for the factor in square brackets in Eq. (12) of OCR).

\section{The effect of heating and cooling}

Let us next examine the effects of altering our treatments of heating and cooling. Figure 11 shows the effect of heating and cooling on the wind structure. The solid line is the reference model. The dashed line is a model where the cooling function $\Lambda$ has been artificially reduced by a factor 1000. The dotted line is a model where the floor temperature is $T_{\text {eff }} / 2$, as opposed to $T_{\text {eff }}$ for the reference model.

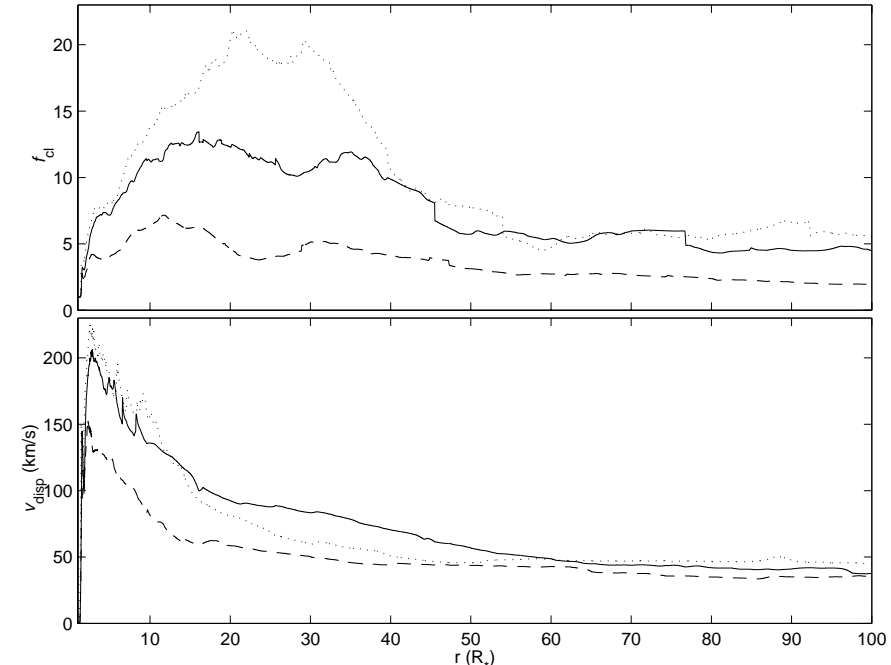

Fig. 11. Effect of heating and cooling on the wind structure. The solid line is the reference model, the dashed line a model where the cooling function $\Lambda$ has been reduced by a factor 1000 , and the dotted line a model with floor temperature equal to $T_{\text {eff }} / 2$.

This can be seen as reducing the effectiveness of radiative heating.

\subsection{Reduced cooling}

In a smooth CAK wind, the maintenance of the wind temperature at values near the stellar effective temperature ensures that there are lots of ions with lines at energies near the peak of the star's radiative flux spectrum. But as gas is heated in shocks or other compressions, the associated ionisation runup means there are generally fewer line transitions with energies that can scatter the star's UV radiative flux. As such, the line-driving force in such hot gas should be substantially reduced.

In our calculations this ionisation runup effect is mimicked by reducing the line-driving opacity of the hot gas by a factor $\exp \left(1-T / T_{\text {floor }}\right)$. When the cooling function is reduced, a much greater fraction of the wind mass can remain above the floor temperature (i.e., $>40 \%$, vs. $<5 \%$ in the reference model). The reduction in line-force associated with the reduced cooling can have a strong effect on the structure; but since the wind outflow is already close to its terminal speed at the radius where such hot structure forms, the effect on the overall bulk outflow is not too great. The influence of reduced cooling on the terminal velocity is barely $10 \%$. The presence of hot gas significantly reduces the amount of structure, however: when a parcel of gas subjected to a positive velocity perturbation is further accelerated due to the instability, it is hindered by the neighbouring hot gas that is not subject to any such instability. This prevents the formation of very strong rarefactions and shocks. Reducing the cooling function therefore produces a wind with almost the same mean flow, but significantly less structure (Fig. 11, dashed line). 


\subsection{Reduced heating}

The effect of reducing the floor temperature is shown by the dotted line in Fig. 11. The model with a reduced floor temperature is more strongly clumped. This can be understood in terms of the lower expansion speed of the dense clumps. Around $5 R_{*}$ both models are equally structured and the cooler floor temperature results in subsequent collisions of narrower clumps than for the reference model, where $T_{\text {floor }}=T_{\text {eff }}$. This also results in a reduced filling-in of the interclump gas, as radiative cooling is less efficient. Eventually though, cooling wins, so the outer wind is at the floor temperature for both models.

Effectively all of the dense clumps (and therefore nearly all of the wind mass, as the clumps comprise most of the wind) are at the floor temperature. It should be emphasised that the assumption of a constant floor temperature is a crude approximation of the effect of radiative heating. If, for some reason, the clumps could shield each other from ionising stellar radiation, the dense gas might become substantially cooler than the $T_{\text {eff }} / 2$ used in this section.

\section{Conclusions}

In this paper we have discussed the structure generated by the instability of the line driving mechanism. We have considered self-excited structure in the sense that we introduce no explicit perturbations at the base of the wind. The emphasis has been on the evolution of structure as it moves out with the flow, described by the radial dependence of statistical quantities such as the clumping factor and the velocity dispersion.

The gas in the outer wind is compressed into a sequence of narrow dense clumps bounded on the starward side by a reverse shock and on the outside by a forward shock. This is the reason why the structure dissipates. The clumpiness of the wind is maintained by collisions between clumps moving at different speeds, not by establishing pressure equilibrium between the clump and its surroundings. This conclusion is robust within the assumptions of this study. A critical assumption in this regard is the floor temperature. Pressure equilibrium could be established if the dense clumps were allowed to cool to very low temperatures. This can only be verified through a detailed investigation of the energy balance in a structured wind, which is a complex problem and is deferred to a later study. The one-dimensionality of the models presented here is bound to be important for clump collisions, as clumps moving at different speeds have to collide, whereas in a multi-dimensional model they can pass each other without colliding.

Clump-clump collisions are also the reason why the strongest density contrasts are formed relatively far from the star (a few tens of stellar radii). Interestingly, this is the region where the millimetre continuum is formed. The results from this study can thus be verified by a comparison of radio and millimetre continuum fluxes.

We have shown that replacing the customary value of $10^{-3} \kappa_{0}$ for the line-strength cut-off by a less artificial value drastically increases the amount of structure. This can be important for i.a. the production of X-rays and further simulations will benefit from imposing a cut-off as close as possible to the realistic value of $\kappa_{0}$

We have also shown that the outer-wind evolution of structure is a pure gasdynamical problem, in the sense that is not affected by external forces. This allows us to consider more economical models for future work.

Acknowledgements. We thank R. Blomme, D. Cohen, A. Feldmeier, K. Gayley, J. Puls and S. Van Loo for many stimulating discussions on line-driving and wind structure. This research was supported in part by NASA grant NAG53530 to the Bartol Research Institute at the University of Delaware. MCR gratefully acknowledges support from ESAProdex project No. 13346/98/NL/VJ(ic).

\section{References}

Abbott, D. H. 1982, ApJ, 259, 282

Abbott, D. H., Bieging, J. H., \& Churchwell, E. 1981, ApJ, 250,645

Bieging, J. H., Abbott, D. H., \& Churchwell, E. 1989, ApJ, 340,518

Castor, J. I., Abbott, D. C., \& Klein, R. I. 1975, ApJ, 195, 157 (CAK)

Chen, W., \& White, R. L. 1994, ApSS, 221, 259

Colella, P., Woodward, P. 1984, J. Comput. Phys., 54, 174

Dessart, L., \& Owocki, S. P., in preparation

Drew, J. A. 1989, ApJS, 71, 267

Feldmeier, A. 1995, A\&A, 299, 523

Feldmeier, A., Kudritzki, R.-P., Palsa, R., Pauldrach, A. W. A., \& Puls, J. 1997a, A\&A, 320, 899

Feldmeier, A., Puls, J., \& Pauldrach, A. W. A. 1997b, A\&A, 322,878

Friend, D., \& Abbott, D. A. 1986, ApJ, 311, 701

Hillier, D. J., Kudritzki, R.-P., Pauldrach, A. W., et al. 1993, A\&A, 276, 117

Gayley, K. G. 1995, ApJ, 454, 410

Grosdidier, Y., Moffat, A. F. J., Joncas, G., \& Acker, A. 1998, ApJ, 506, L127

Heitler W., 1954, The Quantum Theory of Radiation, Third edition (Oxford University Press)

Laney, C. B. 1998, Computational Gasdynamics (Cambridge University Press)

Lucy, L. B. 1982a, ApJ, 255, 278

Lucy, L. B. 1982b, ApJ, 255, 286

Lucy, L. B., \& Solomon, P. M. 1970, ApJ, 159, 879

MacGregor, K., Hartmann, L., \& Raymond, J. 1979, ApJ 251, 514

Milne, E. A. 1926, MNRAS, 86, 459

Owocki, S. P. 1999, IAU Colloq. 169, Variable and Nonspherical Stellar Winds in Luminous Hot Stars, ed. B. Wolf, A. Fullerton, \& O. Stahl, (Springer Lecture Notes in Physics), 523, 294 
Owocki, S. P., \& Puls, J. 1996, ApJ, 462, 894 (OP96)

Owocki, S. P., \& Puls, J. 1999, ApJ, 510, 355 (OP99)

Owocki, S. P., \& Rybicki, G. B. 1984, ApJ, 286, 337

Owocki, S. P., \& Rybicki, G. B. 1985, ApJ, 299, 265

Owocki, S. P., Castor, J. I., \& Rybicki, G. B. 1988, ApJ, 335, 914 (OCR)

Owocki, S. P., Runacres, M. C., \& Cohen, D. H. 2000, in ASP

Conf. Ser. 204, Thermal and Ionization Aspects of Flows

from Hot Stars, ed. H. J. G. L. M Lamers, \& A. Sapar, 183

Pauldrach, A., Puls, J., \& Kudritzki, R. P. 1986, A\&A 164, 86

Poe, C. H., Owocki, S. P., \& Castor, J. I., ApJ 358, 199
Pollock, A. M. T. 1987, A\&A, 171, 135

Prinja, R. K. 1998, in Cyclical Variability in Stellar Winds, ed. L. Kaper \& A. W. Fullerton (Garching: ESO)

Raymond, J. C., Cox, D. P., \& Smith, B. W. 1976, ApJ, 204, 290

Rybicki, G. B., Owocki, S. P., \& Castor, J. I. 1990, ApJ, 349, 274

Wright, A. E., \& Barlow, M. J. 1975, MNRAS, 170, 41

Zel'dovich, Y. B., \& Raizer, Y. P. 1967, Physics of Shock Waves and High-Temperature Hydrodynamic Phenomena, vol. I (Academic Press, New York) 